\title{
Parental smoking during pregnancy and risk of overweight and obesity in the daughter
}

\author{
Holly R. Harris, Sc.D. ${ }^{1,2}$, Walter C. Willett, M.D., Dr.P.H. ${ }^{3,4,5}$, and Karin B. Michels, Sc.D., \\ Ph.D. ${ }^{1,3,5}$ \\ ${ }^{1}$ Obstetrics and Gynecology Epidemiology Center, Department of Obstetrics, Gynecology, and \\ Reproductive Biology, Brigham and Women's Hospital, Harvard Medical School, Boston, \\ Massachusetts, U.S.A. \\ ${ }^{2}$ Division of Nutritional Epidemiology, The National Institute for Environmental Medicine, \\ Karolinska Institutet, Stockholm, Sweden \\ ${ }^{3}$ Department of Epidemiology, Harvard School of Public Health, Boston, Massachusetts, U.S.A. \\ ${ }^{4}$ Department of Nutrition, Harvard School of Public Health, Boston, Massachusetts, U.S.A. \\ ${ }^{5}$ Channing Division of Network Medicine, Department of Medicine, Brigham and Women's \\ Hospital and Harvard Medical School, Boston, Massachusetts, U.S.A.
}

\section{Abstract \\ Objective-Emerging evidence suggests that prenatal exposures may affect long-term health outcomes. In utero exposure to smoking is associated with an increased risk of overweight and obesity in children and adolescents. However, few studies have examined how prenatal exposure to parental smoking influences risk of obesity in adulthood and whether these associations are independent of childhood and adolescent adiposity. The aim of the current study was to investigate whether prenatal exposure to parental smoking influences body size in adulthood and whether any association may be mediated by childhood and adolescent body size.}

Methods-We investigated the association between parental smoking during pregnancy and risk of overweight and obesity in adulthood and at age 18, and adiposity during childhood among 35,370 participants in the Nurses' Health Study II. Data on smoking during pregnancy and socioeconomic variables were provided by the mothers, and anthropometric data and adult risk factors were reported by participants.

Results-After adjustment for socioeconomic and behavioral variables, maternal smoking during pregnancy was associated with adiposity at ages 5-10, age 18, and during adulthood. For age 18 overweight the ORs (95\% CIs) for 1-14, 15-24, and 25+cigarettes/day were 1.13 (1.18$1.50), 1.40(1.20-1.64)$, and $1.15(0.79-1.69)$ and for obesity were 1.41 (1.14-1.75), 1.69 (1.312.18 ), and 2.36 (1.44-3.86). The corresponding ORs (95\% CIs) for obesity in adulthood were 1.26

\footnotetext{
Users may view, print, copy, download and text and data- mine the content in such documents, for the purposes of academic research, subject always to the full Conditions of use: http://www.nature.com/authors/editorial_policies/license.html\#terms

Corresponding author and request for reprints: Dr. Holly R. Harris, Ob/Gyn Epidemiology Center, Brigham and Women's Hospital, 221 Longwood Avenue, Boston, MA 02115 hharris3@partners.org; phone: 617-525-8093; fax: 617-732-4899).

Conflict of interest: The authors declare no conflicts of interest.
} 
(1.16-1.37), 1.46 (1.30-1.63), and 1.43 (1.10-1.86). Risk of adiposity was not increased among daughters whose mothers stopped smoking during the first trimester $(\mathrm{OR}[95 \% \mathrm{CI}]$ for overweight (1.03 [95\% CI 0.90-1.17] and obesity (1.12 [95\% CI 0.97-1.30]). Women whose fathers smoked during pregnancy were also at increased risk of overweight and obesity in adulthood with covariate-adjusted ORs (95\% CIs) for obesity of 1.19 (1.11-1.29) for 1-14 cigarettes/day, 1.27 (1.18-1.37) for 15-24 cigarettes/day, and 1.40 (1.27-1.54) for 25+ cigarettes/day compared to fathers who did not smoke ( $\mathrm{p}_{\text {trend }}<0.0001$ ). Paternal smoking during pregnancy was also associated with an increased risk of obesity at age 18 among those whose fathers smoked 15 or more cigarettes/day but was not associated with childhood body size.

Conclusions-Maternal smoking during pregnancy was associated in a dose-response manner with overweight and obesity in the daughter through adolescence and adult life. Smoking cessation during the first trimester appears to mitigate this excess risk.

Paternal smoking was also associated with risk of overweight and obesity of the adult daughter and this association persisted after adjustment for maternal smoking.

\section{Keywords}

pregnancy; prenatal programming; cigarette smoking; obesity

\section{Introduction}

Emerging evidence suggests that prenatal exposures may affect long-term health outcomes. The developmental origins of health and disease hypothesis (DOHAD) proposes that exposures before birth may result in persistent adaptations including alterations in metabolism. ${ }^{1}$ Children exposed to cigarette smoking in utero have a lower birthweight compared to children of non-smokers ${ }^{2,3}$ and conversely an increased risk of overweight and obesity as children and young adults. ${ }^{4-10}$ A 2008 meta-analysis reported a 50\% increased risk of overweight in children whose mothers smoked during pregnancy compared to children of non-smoking mothers. ${ }^{8}$ More recently, Durmus, et al. reported an increased risk of obesity at age 4 among children whose mothers smoked during pregnancy but did not observe an association with paternal smoking among non-smoking mothers. ${ }^{5}$ To our knowledge, only two studies have examined the relation of prenatal exposure to smoking to body size in later adult life. The authors of these studies reported that exposure to maternal smoking in utero was associated with a $34-41 \%$ increased risk of adult obesity, ${ }^{11,12}$ however neither reported adjustment for body size in early life.

While previous studies have suggested that the association between maternal smoking and body size in childhood and young adulthood is independent of birthweight ${ }^{4,8-10,13-16}$ it is unclear whether any association between maternal smoking and later life adult body mass index (BMI) may be mediated by childhood and adolescent body size. In addition, few studies have examined the influence of paternal smoking on body size throughout the lifecourse. The aim of the current study was to investigate whether prenatal exposure to parental smoking influences body size in adulthood and whether any association may be mediated by childhood and adolescent body size. In addition, we investigated whether these 
associations were accounted for by parental BMI or other determinants of adiposity including physical activity and diet.

\section{Methods}

\section{Study population}

The Nurses' Health Study II (NHS II) was established in 1989 when 116,430 female nurses completed a questionnaire about lifestyle factors, anthropometric variables, and prevalent disease. Follow-up questionnaires were sent biennially to collect updated information.

In 2001, mothers of NHS II daughters were invited to complete a questionnaire regarding their daughter. Details about the Nurses' Mothers' Cohort have been provided elsewhere. ${ }^{17}$ Information on pregnancy and early life exposures were obtained for 35,794 participants (76.5\% response rate). Women were excluded from the current analyses if they had been adopted ( $\mathrm{n}=113)$, were missing information on maternal smoking $(\mathrm{n}=122)$, or were missing BMI in 1989 (n=189). Implied consent was assumed upon return of the completed questionnaire. This study was approved by the Institutional Review Boards of the Harvard School of Public Health and Brigham and Women's Hospital.

\section{Assessment of smoking}

Mothers reported whether they smoked during pregnancy with their daughter, the number of cigarettes smoked per day, whether they stopped smoking during pregnancy, and if so when they stopped. The validity of recalled maternal smoking during pregnancy was found to be high in the National Collaborative Perinatal Project (sensitivity $=0.86$, specificity=0.94). ${ }^{18}$ Mothers also reported whether the nurse's father smoked and if so when he smoked, and the number of cigarettes smoked per day.

\section{Assessment of body size}

NHS II participants reported their height, weight, and weight at age 18 at baseline (1989); current weight was updated on biennial questionnaires. BMI in 2007 was used for the main analysis. In 1989 participants were asked to recall their body shape at ages 5 and 10 using a 9-level figure drawing. ${ }^{19}$ The validity of recalled body shape using this drawing was assessed in the Third Harvard Growth Study. Body shape recalled by 181 participants between ages 71-76 years was compared to BMI taken as part of annual examinations during childhood. Pearson correlations were 0.60 and 0.70 , for age 5 and 10 years, respectively. ${ }^{20}$

\section{Covariate assessment}

NHS II participants reported their age, year of birth, age at menarche, husband's education, income, physical activity, caloric intake, alcohol consumption, smoking status, parity, and age at first birth on baseline and follow-up questionnaires. Nurses' mothers reported maternal and paternal age at time of the nurse daughter's birth, maternal and paternal education level, maternal weight gain during pregnancy, maternal prepregnancy weight, paternal weight at the time of the nurse daughter's birth, maternal and paternal height, maternal and paternal history of diabetes, maternal and paternal occupation, parents' home 
ownership at the time of the nurse daughter's birth, the nurse's birthweight, gestational age, and breast feeding history on the Mothers' questionnaire.

\section{Statistical analyses}

Polytomous logistic regression was used to estimate odds ratios (OR) and 95\% confidence intervals (CIs) for the associations between maternal and paternal smoking during pregnancy and BMI in adulthood, BMI at age 18, and childhood body shape. Maternal smoking was categorized into 'No smoking during pregnancy', 'Quit smoking during pregnancy', '1-14 cigarettes/day', '15-24 cigarettes/day', and '25+/cigarettes/day'. Paternal smoking was categorized into 'Non-smoker', '1-14/day', '15-24/day', and '25+/day'. Secondary analyses were conducted by combined parental smoking (no smoking from either parent, father smoker/mother non-smoker, father non-smoker/mother smoker, both smokers).

BMI $\left(\mathrm{kg} / \mathrm{m}^{2}\right)$ was categorized as: $<25,25-<30$ (overweight), and $>30$ (obese). Somatotypes at ages 5 and 10 were averaged to obtain estimates of childhood body size and categorized as $1-2,2.5-3,3.5-4$, and $\geq 4.5$.

We considered 2 main covariate-adjusted models; the first adjusted for parental and nurse's prenatal characteristics and the second adjusted for parental and all nurse characteristics. We included the following a priori potential confounders in the parental and nurse's prenatal characteristics model: age of the nurse, birth year, maternal and paternal age at birth, maternal and paternal education, maternal and paternal history of diabetes, maternal and paternal occupation, maternal weight gain during pregnancy, maternal prepregnancy BMI, paternal BMI, and home ownership at birth. In addition the following a priori potential confounders were included in the parental and all nurse characteristics model which represents the final model: gestational age, breast feeding history in infancy, age at menarche, parity/age at first birth, nurse's smoking status, alcohol intake, caloric intake, physical activity, dietary change score, ${ }^{21}$ income, and nurse's husband's education. Models with maternal smoking were also simultaneously adjusted for paternal smoking and vice versa. We also explored the role of birthweight, childhood body size, and BMI at age 18 as potential mediators by adding them to the models with adult BMI as the outcome, and added birthweight and childhood body size to the models with BMI at age 18 as the outcome. In addition, adolescent smoking status, alcohol intake, caloric intake, and physical activity were included in the BMI at age 18 analyses. Tests for linear trend were performed by assigning the category midpoints to each participant in that group.

We examined whether the association between maternal smoking and body size varied by maternal prepregnancy BMI, weight gain during pregnancy and birthweight. Effect modification was assessed with a likelihood ratio test comparing a model with the crossproduct term between maternal smoking and each potential effect modifier to the model with main effects. Chi-square tests were used to obtain p-values for the likelihood ratio test statistics. All tests of statistical significance were two sided and all statistical analyses were performed using SAS Version 9.2 (SAS Institute Inc., Cary, NC). 


\section{Results}

Among 35,370 women included in this study, 9,245 (26.1\%) had mothers who reported smoking during pregnancy. The mean (standard deviation [SD]) adult BMI in 2007 among daughters of smokers was 27.4 (6.3) compared to 27.1 (6.1) for daughters of non-smokers while the daughters of smokers had a lower mean birthweight (grams) (SD) than the nonsmokers, 3158 (513) and 3335 (503), respectively. Mothers who smoked during pregnancy had a lower prepregnancy BMI, were more likely to have a college education, and less likely to own their own home at the time of their daughter's birth compared to those who did not smoke (Table 1).

We observed a statistically significant association between maternal smoking during pregnancy and overweight and obesity in adulthood with the risk increasing with the number of cigarettes smoked daily. Compared to women of mothers who did not smoke during pregnancy the maternal, paternal, and nurse characteristics-adjusted ORs (95\% CIs) for overweight for women whose mothers smoked during pregnancy were $1.10(1.02-1.19)$ for 1-14 cigarettes/day, $1.20(1.08-1.33)$ for 15-24 cigarettes/day, and $1.35(1.07-1.72)$ for $25+$ cigarettes/day ( $\left.\mathrm{p}_{\text {trend }}<0.0001\right)$. The corresponding ORs (95\% CIs) for obesity were 1.26 (1.16-1.37), 1.46 (1.30-1.63), and 1.43 (1.10-1.86) ( $\left.\mathrm{p}_{\text {trend }}<0.0001\right)$ (Table 2). Adjustment for paternal smoking (Table 2) and body size in childhood (data not shown) slightly attenuated the associations but they remained significant. Adjustment for BMI at age 18 attenuated the associations but the trends remained significant (Table 2). When highly correlated variables are included together in a regression model this may cause an inflation of the standard errors which may affect the precision of the estimate. We did not observe an inflation of the standard errors when BMI at age 18 was included in a model simultaneously with adult BMI as the observed correlation between BMI at age 18 and adult BMI (measured in 2007) was only 0.53 ( $\mathrm{p}<0.0001$ level) and this is well below the threshold of 0.9 above which collinearity may be a problem. ${ }^{22}$ Results were similar when adult BMI from questionnaires before 2007 were used (data not shown).

Fifteen percent $(n=1,385)$ of women who smoked during pregnancy reported quitting $(1,141$ first trimester, 135 second trimester, and 109 third trimester). Among the daughters of the women who quit smoking there was no association with overweight (1.03 [95\% CI 0.901.17]) and the association with obesity was not significant (1.12 [95\% CI 0.97-1.30]). When quitting was examined by trimester the ORs (95\% CIs) for obesity were $1.13(0.96-1.33)$ for the first trimester, $1.23(0.79-1.92)$ for the second trimester, and $0.94(0.56-1.60)$ for the third trimester. However, the results for the second and third trimesters were based on small numbers ( $\mathrm{n}=44$ and 24 , respectively).

An association between maternal smoking during pregnancy and BMI at age 18 and body size in childhood was also observed. Compared to women of mothers who did not smoke during pregnancy women of mothers who smoked during pregnancy had covariate-adjusted ORs (95\% CIs) for overweight at age 18 of $1.33(1.18-1.50)$ for $1-14$ cigarettes/day, 1.40 (1.20-1.64) for 15-24 cigarettes/day, and $1.15(0.79-1.69)$ for $25+$ cigarettes/day $\left(\mathrm{p}_{\text {trend }}<0.0001\right)$. The corresponding ORs (95\% CIs) for obesity were 1.41 (1.14-1.75), 1.69 $(1.31-2.18)$, and $2.36(1.44-3.86)\left(\mathrm{p}_{\text {trend }}<0.0001\right)$. This association was slightly attenuated 
after adjustment for body size in childhood (Table 3). In addition, the daughters of mothers who smoked during pregnancy were more likely to report increased childhood body size (Table 4).

Women whose fathers smoked during pregnancy were at increased risk of overweight and obesity in adulthood with covariate-adjusted ORs (95\% CIs) for overweight of 1.11 (1.031.18) for 1-14 cigarettes/day, 1.14 (1.07-1.22) for 15-24 cigarettes/day, and 1.19 (1.09$1.31)$ for $25+$ cigarettes/day compared to fathers who did not smoke ( $\left.\mathrm{p}_{\text {trend }}<0.0001\right)$. The corresponding ORs (95\% CIs) for obesity were 1.19 (1.11-1.29), 1.27 (1.18-1.37), and 1.40 $(1.27-1.54)\left(\mathrm{p}_{\text {trend }}<0.0001\right)$ (Table 5). An attenuated but still statistically significant association remained when paternal smoking was examined among non-smoking mothers (data not shown). The strongest association with adult overweight and obesity was observed when both parents smoked (ORs [95\% CIs] for adult BMI of 1.22 [95\% CI 1.13-1.31] for overweight and 1.49 [95\% CI 1.37-1.61] for obesity.

Paternal smoking during pregnancy was associated with an increased risk of obesity at age 18 among those whose fathers smoked 15 or more cigarettes/day (ORs [95\% CIs] of 1.22 [95\% CI 1.00-1.49] for 15-24 cigarettes/day and 1.52 [95\% CI 1.21-1.92] for 25+ cigarettes/day). When paternal smoking was examined among non-smoking mothers only the association with obesity among those whose fathers smoked 25+ cigarettes/day remained significant (1.39 [1.02-1.88]). Paternal smoking was not associated with childhood body size (data not shown). No significant interactions were observed for any of the body size outcomes by maternal prepregnancy BMI, weight gain during pregnancy, or birthweight.

\section{Discussion}

In this large cohort study, maternal smoking during pregnancy was independently associated in a dose-response manner with increased risk of elevated body mass during childhood, adolescence, and adulthood in the daughter. This association was strongest among daughters of women who smoked 25 or more cigarettes per day during pregnancy. Paternal smoking was also associated with risk of overweight and obesity of the adult daughter and this association persisted after adjustment for maternal smoking. Most studies examining in utero exposure to maternal cigarette smoking have been limited to examining body size outcomes in childhood and adolescence and have consistently reported increased risk of overweight and obesity among exposed children. ${ }^{8}$ Few studies have examined the association with body size in later adult life. Consistent with our results for adult BMI, Power and Jefferis reported an adjusted OR (95\%) of 1.45 (1.13-1.87) for the association between maternal smoking and obesity in 2921 women 33 years of age ${ }^{11}$. Among 4917 participants (age 33 years) from the British National Child Development Study, Montgomery and Ekbom reported ORs (95\% CIs) for obesity of 1.34 (1.07-1.60) for medium smokers (1-9 cigarettes/day) and $1.38(1.06-1.79)$ for heavy smokers (>10 cigarettes/day). ${ }^{12}$ Neither of these studies reported adjustment for body size in childhood or adolescence. In addition, they did not have the breadth of potential confounders that were available in our study including maternal weight gain during pregnancy and adult diet and physical activity in the daughter. In our analysis adjustment for BMI at age 18 attenuated the associations but a significant trend remained suggesting that at least part of the effect of 
maternal smoking on adult BMI may be independent of adolescent body size. Consistent with prior studies we found only marginal changes in the associations when adjusting for differences in birthweight, a potential intermediate. ${ }^{4,} 8-10,13-16$

Tobacco smoke contains over 4,000 different chemicals making it difficult to elucidate the mechanisms through which exposure to smoking in utero may increase body size in childhood, adolescence, and adulthood. Rats prenatally exposed to nicotine have increased body fat ${ }^{23}$ and weight ${ }^{24}$ making nicotine a possible candidate. Nicotine may influence body size through the programming of the fetal hypothalamic-pituitary axis ${ }^{25}$ or alterations in pancreatic function ${ }^{26}$ and appetite control. ${ }^{27}$ We observed an association between both maternal and paternal smoking and adult BMI but did not observe associations between paternal smoking and child body size. This is consistent with results from the Generation $\mathrm{R}$ Study of children in the Netherlands where a significant association was observed between maternal smoking and childhood obesity while no association was observed between paternal smoking and childhood obesity among non-smoking mothers. ${ }^{5}$ This may suggest different mechanisms through which in utero exposure to maternal smoking versus exposure to paternal second hand smoke (SHS) influences adult and early life body size. The significant effect estimates observed for maternal smoking but not paternal smoking for childhood body size may suggest a direct intrauterine effect of maternal smoking on childhood body size. Alternatively, it could indicate that exposure in childhood to parental SHS is being captured by the paternal smoking variable since $93 \%$ of fathers in our study smoked both during pregnancy and early childhood. This SHS exposure during the daughter's childhood could influence adult adiposity as SHS exposure in adults and adolescents has been associated with insulin resistance, metabolic syndrome, and type II diabetes. ${ }^{28-31}$ In addition, SHS exposure during childhood has been associated with insulin resistance at the age of 10 years. ${ }^{32}$ Thus, SHS exposure during childhood may cause alterations in metabolism that become most apparent during adulthood. The mothers in our study were not asked about their smoking habits during their daughter's early childhood but it is likely that most women who smoked during pregnancy did not quit immediately following pregnancy. Finally, the stronger effect estimates observed for maternal smoking compared to paternal smoking for childhood adiposity may suggest a direct intrauterine effect of maternal smoking on childhood body size.

Parental smoking during pregnancy was recalled by nurses' mothers, and body shape in childhood, and BMI at age 18 were recalled by the nurse participants, which may have resulted in misclassification of these variables. In addition, self-reported weight and height used to calculate BMI may also be affected by measurement error. However, long-term maternal recall of smoking during pregnancy has been found to be highly accurate. ${ }^{18}$ Also, recall of body shape using the 9-level figure diagram is correlated with BMI at the same age ${ }^{20}$ however, error in the classification of childhood body size could have resulted in incomplete adjustment for this variable in the models where childhood body size was considered as a potential mediator. Finally, correlations of 0.84 and higher have been reported for recalled weight and height at age 18 and self-reported weight compared to direct measures of these variables. ${ }^{33,34}$ Confounding by socioeconomic and lifestyle factors is often a concern when smoking is examined. However, the participants in this study were 
born between 1945 and 1964 corresponding to a time when information on the harmful effects of tobacco use was just beginning to reach the public. Per capita consumption of cigarettes peaked in the U.S in $1963^{35}$ and in 1964 the U.S. Surgeon General issued a report highlighting the dangers of cigarette smoking. Thus our results are less likely to be due to confounding by health-related behaviors than analyses examining effects of smoking in more recent years. In addition, we adjusted for many potential confounders including parental education, occupation, and BMI, and the nurse's income, physical activity, diet, and smoking status. If anything, adjustment for these variables slightly increased the strength of the association with maternal smoking, suggesting that confounding by unmeasured factors is unlikely to account for our findings.

Our study has several strengths. To our knowledge, this is the largest study to examine the association between parental smoking and overweight and obesity in adulthood. We had detailed information on maternal and paternal smoking habits including the number of cigarettes smoked and if the mother quit smoking during pregnancy which allowed us to examine the dose-response relation. In addition, we have anthropometric data from across the lifecourse (birthweight, childhood body size, BMI at age 18, and multiple assessments of adult BMI), which allowed us to examine independent associations with each of these outcomes. We also have high follow-up rates and data on many covariates that have been collected and updated at two-year intervals.

In conclusion, our findings suggest that maternal and paternal smoking during pregnancy increases the risk of overweight and obesity in the offspring during adulthood in a doseresponse manner. These associations appear to be independent of childhood and adolescent body size and birthweight. In addition, maternal smoking, but not paternal smoking, was associated with increased body size in childhood and adolescence. Future studies that can separate the effects of parental smoking exposure in utero and early childhood may further our understanding of the importance of the timing of smoking exposure as well as the mechanisms behind these associations.

\section{Acknowledgments}

Funding: The Nurses' Health Study II is supported by Public Health Service grant CA50385 from the National Cancer Institute, National Institutes of Health, U.S. Department of Health and Human Services. The Nurses' Mothers' Cohort Study was funded by the Intramural Research Program of the National Cancer Institute research contract N02-RC-17027, and by PO 263 MQ 411027 from the National Cancer Institute. H.R.H. was supported by the NIH training grant T32 ES007069 and MCHB grant number 5T76MC00001 (formerly MCJ201). The funders has no role in study design, data collection and analysis, decision to publish, or preparation of the manuscript.

\section{References}

1. Barker D. Fetal origins of coronary heart disease. BMJ. 1995; 311(6998):171-174. [PubMed: 7613432]

2. Andres R, Day M. Perinatal complications associated with maternal tobacco use. Semin Neonatol. 2000; 5(3):231-241. [PubMed: 10956448]

3. Cnattingius S. The epidemiology of smoking during pregnancy: Smoking prevalence, maternal characteristics, and pregnancy outcomes. Nicotine Tob Res. 2004; 6(Suppl 2):S125-S140.

[PubMed: 15203816] 
4. Beyerlein A, Rückinger S, Toschke A, Schaffrath Rosario A, von Kries R. Is low birthweight in the causal pathway of the association between maternal smoking in pregnancy and higher BMI in the offspring? Eur J Epidemiol. 2011; 26(5):413-420. [PubMed: 21360298]

5. Durmuş B, Kruithof CJ, Gillman MH, Willemsen SP, Hofman A, Raat H, et al. Parental smoking during pregnancy, early growth, and risk of obesity in preschool children: the Generation R Study. Am J Clin Nutr. 2011; 94(1):164-171. [PubMed: 21593510]

6. Ino T. Maternal smoking during pregnancy and offspring obesity: Meta-analysis. Pediatr Int. 2010; 52(1):94-99. [PubMed: 19400912]

7. Koshy G, Delpisheh A, Brabin BJ. Dose response association of pregnancy cigarette smoke exposure, childhood stature, overweight and obesity. Eur J Public Health. 2011; 21(3):286-291. [PubMed: 21126981]

8. Oken E, Levitan E, Gillman M. Maternal smoking during pregnancy and child overweight: systematic review and meta-analysis. Int J Obes. 2008; 32(2):201-210.

9. Koupil I, Toivanen P. Social and early-life determinants of overweight and obesity in 18-year-old Swedish men. Int J Obes. 2008; 32(1):73-81.

10. Mamun A, O'Callaghan M, Williams G, Najman J. Maternal Smoking During Pregnancy Predicts Adult Offspring Cardiovascular Risk Factors - Evidence from a Community-Based Large Birth Cohort Study. PLoS ONE. 2012; 7(7):e41106. [PubMed: 22829913]

11. Power C, Jefferis BJ. Fetal environment and subsequent obesity: a study of maternal smoking. Int J Epidemiol. 2002; 31(2):413-419. [PubMed: 11980805]

12. Montgomery S, Ekbom A. Smoking during pregnancy and diabetes mellitus in a British longitudinal cohort. BMJ. 2002; 324(7328):26-27. [PubMed: 11777801]

13. Leary SD, Smith GD, Rogers IS, Reilly JJ, Wells JCK, Ness AR. Smoking during Pregnancy and Offspring Fat and Lean Mass in Childhood[ast]. Obesity. 2006; 14(12):2284-2293. [PubMed: 17189557]

14. Oken E, Huh S, Taveras E, Rich-Edwards J, Gillman M. Associations of maternal prenatal smoking with child adiposity and blood pressure. Obes Res. 2005; 13(11):2021-2028. [PubMed: 16339135]

15. Toschke AM, Koletzko B, Slikker WJ, Hermann M, von Kries R. Childhood obesity is associated with maternal smoking in pregnancy. Eur J Pediatr. 2002; 161(8):445-448. [PubMed: 12172830]

16. Widerøe M, Vik T, Jacobsen G, Bakketeig LS. Does maternal smoking during pregnancy cause childhood overweight? Paediatr Perinat Epidemiol. 2003; 17(2):171-179. [PubMed: 12675784]

17. Michels K, Willett W, Graubard B, Vaidya R, Cantwell M, Sansbury L, et al. A longitudinal study of infant feeding and obesity throughout the lifecourse. Int J Obes. 2007; 31(7):1078-1085.

18. Tomeo CA, Rich-Edwards JW, Michels KB, Berkey CS, Hunter DJ, Frazier AL, et al. Reproducibility and Validity of Maternal Recall of Pregnancy-Related Events. Epidemiology. 1999; 10(6):774-777. [PubMed: 10535796]

19. Stunkard, A.; Sorensen, T.; Schulsinger, F. Use of the Danish Adoption Register for the study of obesity and thinness. In: Kety, SRL.; Sideman, S., et al., editors. The Genetics of Neurological and Psychiatric Disorders. New York City: Raven Press; 1983.

20. Must A, Willett W, Dietz W. Remote Recall of Childhood Height, Weight, and Body Build by Elderly Subjects. Am J Epidemiol. 1993; 138(1):56-64. [PubMed: 8333427]

21. Mozaffarian D, Hao T, Rimm EB, Willett WC, Hu FB. Changes in Diet and Lifestyle and LongTerm Weight Gain in Women and Men. N Engl J Med. 2011; 364(25):2392-2404. [PubMed: 21696306]

22. Kleinbaum, D.; Kupper, L.; Muller, K.; Nizam, A. Applied Regression Analysis and Other Multivariable Methods. 3 edn. Pacific Grove: Duxbury Press; 1998.

23. Gao Y, Holloway A, Zeng Z, Lim G, Petrik J, Foster W, et al. Prenatal exposure to nicotine causes postnatal obesity and altered perivascular adipose tissue function. Obes Res. 2005; 13(4):687-692. [PubMed: 15897477]

24. Newman M, Shytle R, Sanberg P. Locomotor behavioral effects of prenatal and postnatal nicotine exposure in rat off spring. Behav Pharmacol. 1999; 10(6-7):699-706. [PubMed: 10780512] 
25. McDonald SD, Walker M, Perkins SL, Beyene J, Murphy K, Gibb W, et al. The effect of tobacco exposure on the fetal hypothalamic-pituitary-adrenal axis. BJOG. 2006; 113(11):1289-1295. [PubMed: 17014678]

26. Bruin JE, Gerstein HC, Morrison KM, Holloway AC. Increased Pancreatic Beta-Cell Apoptosis following Fetal and Neonatal Exposure to Nicotine Is Mediated via the Mitochondria. Toxicol Sci. 2008; 103(2):362-370. [PubMed: 18203686]

27. Oken E, Gillman M. Fetal origins of obesity. Obes Res. 2003; 11(4):496-506. [PubMed: 12690076]

28. Henkin L, Zaccaro D, Haffner S, Karter A, Rewers M, Sholinsky P, et al. Cigarette Smoking, Environmental Tobacco Smoke Exposure and Insulin Sensitivity: The Insulin Resistance Atherosclerosis Study. Ann Epidemiol. 1999; 9(5):290-296. [PubMed: 10976855]

29. Houston TK, Person SD, Pletcher MJ, Liu K, Iribarren C, Kiefe CI. Active and passive smoking and development of glucose intolerance among young adults in a prospective cohort: CARDIA study. BMJ. 2006; 332(7549):1064-1069. [PubMed: 16603565]

30. Weitzman M, Cook S, Auinger P, Florin TA, Daniels S, Nguyen M, et al. Tobacco Smoke Exposure Is Associated With the Metabolic Syndrome in Adolescents. Circulation. 2005; 112(6): 862-869. [PubMed: 16061737]

31. Kowall B, Rathmann W, Strassburger K, Heier M, Holle R, Thorand B, et al. Association of passive and active smoking with incident type 2 diabetes mellitus in the elderly population: the KORA S4/F4 cohort study. Eur J Epidemiol. 2010; 25(6):393-402. [PubMed: 20369275]

32. Thiering E, Brüske I, Kratzsch J, Thiery J, Sausenthaler S, Meisinger C, et al. Prenatal and postnatal tobacco smoke exposure and development of insulin resistance in 10 year old children. Int J Hyg Environ Health. 2011; 214(5):361-368. [PubMed: 21570350]

33. Troy L, Hunter D, Manson J, Coldtiz G, Stampfer M, Willett W. The validity of recalled weight among younger women. Int J Obes Relat Metab Disord. 1995; 19(8):570-572. [PubMed: 7489028]

34. Willett, WC. Nutritional Epidemiology. 2 edn. Oxford: Oxford University Press; 1998.

35. Shopland D, Pechacek T, Cullen J. Toward a tobacco-free society. Semin Oncol. 1990; 17(4):402412. [PubMed: 2201086] 


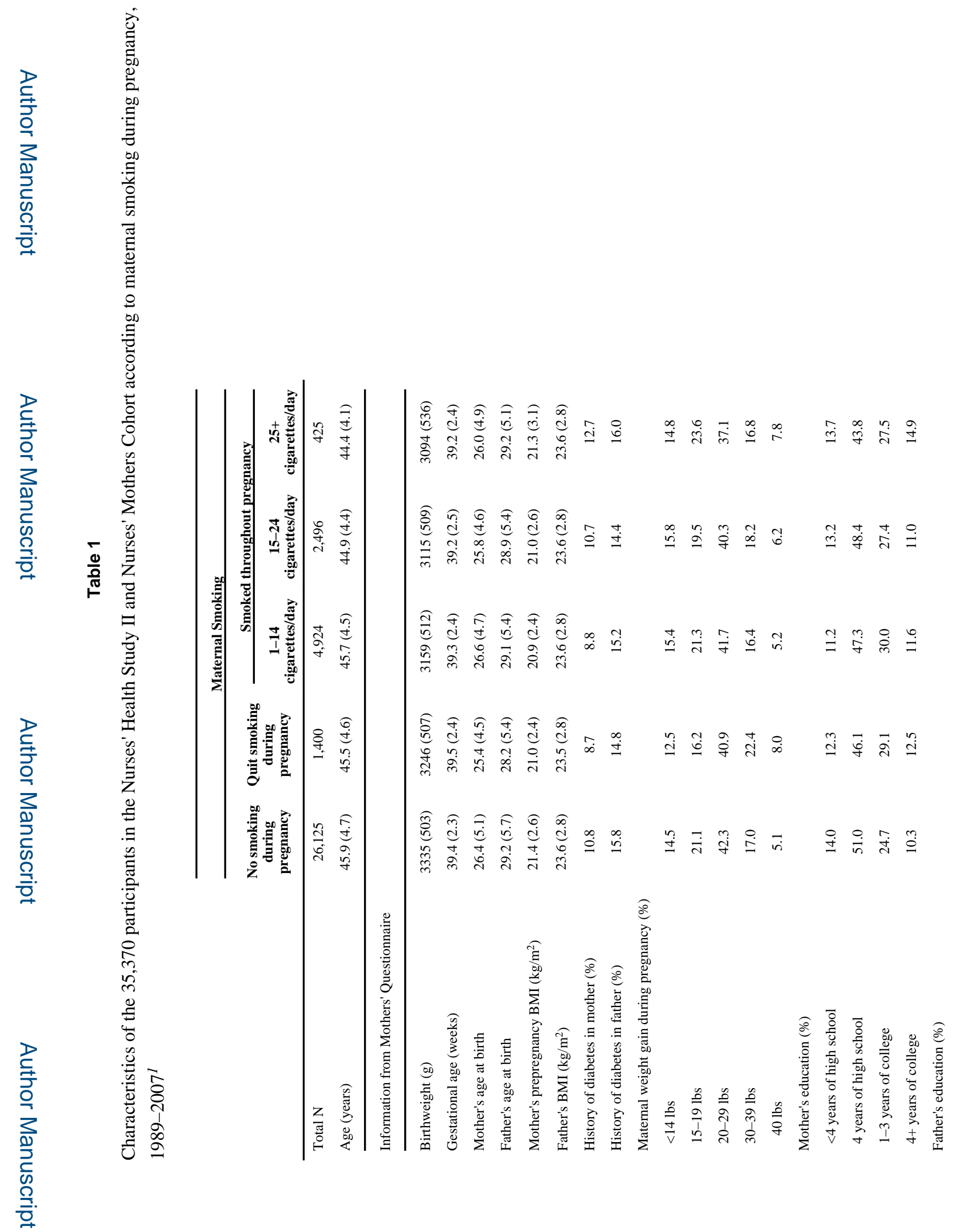

Int J Obes (Lond). Author manuscript; available in PMC 2014 April 01. 
Harris et al.

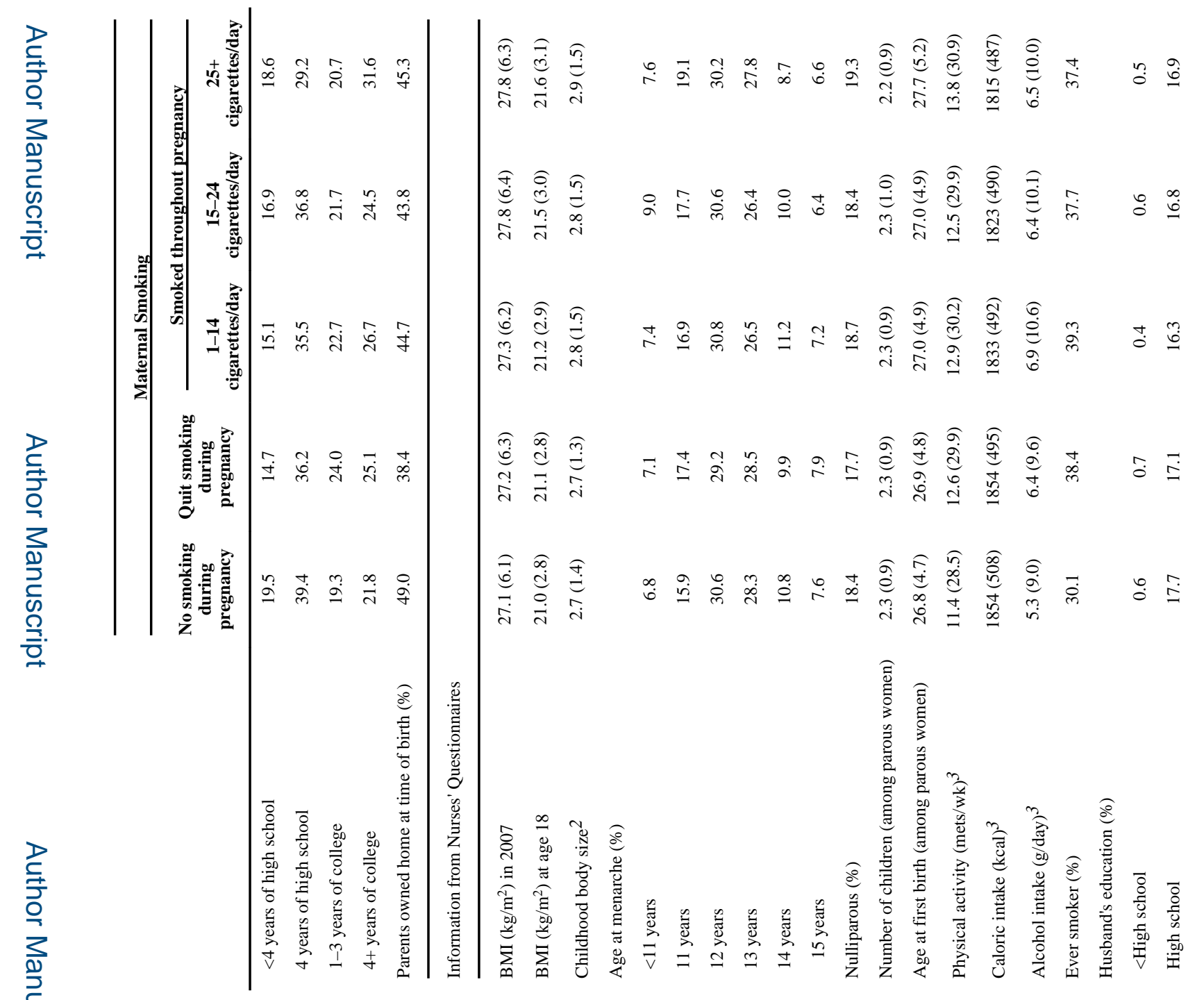

Int J Obes (Lond). Author manuscript; available in PMC 2014 April 01. 

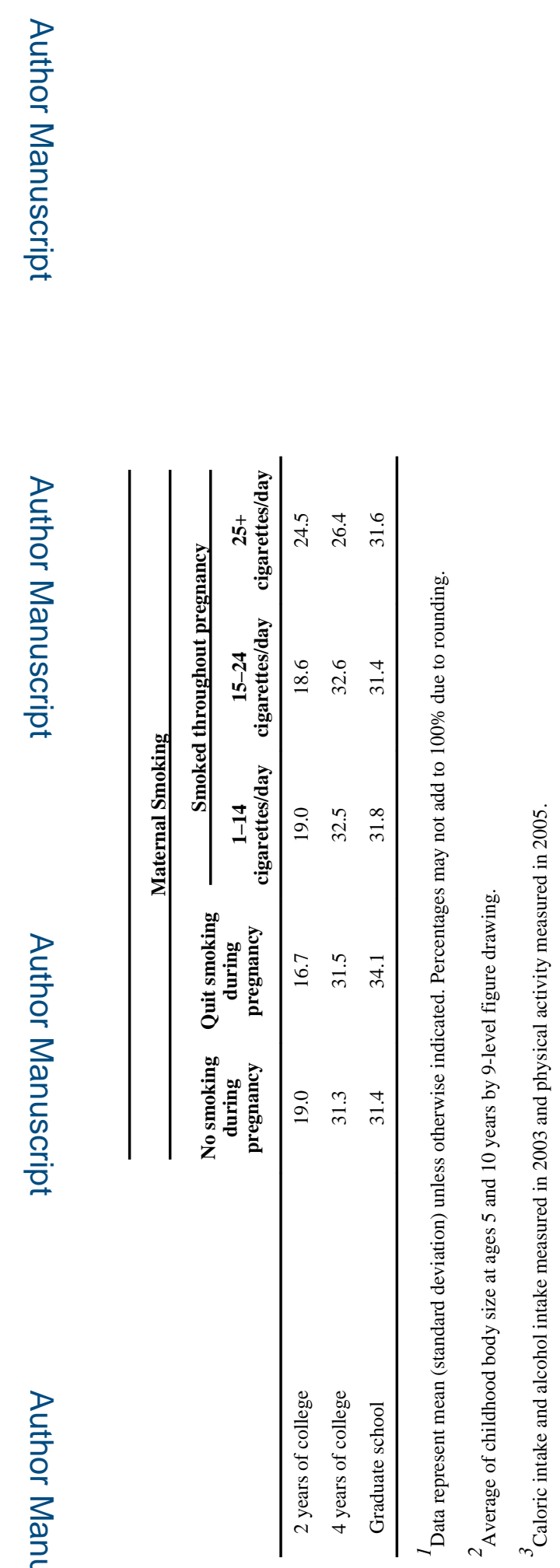

Int J Obes (Lond). Author manuscript; available in PMC 2014 April 01 


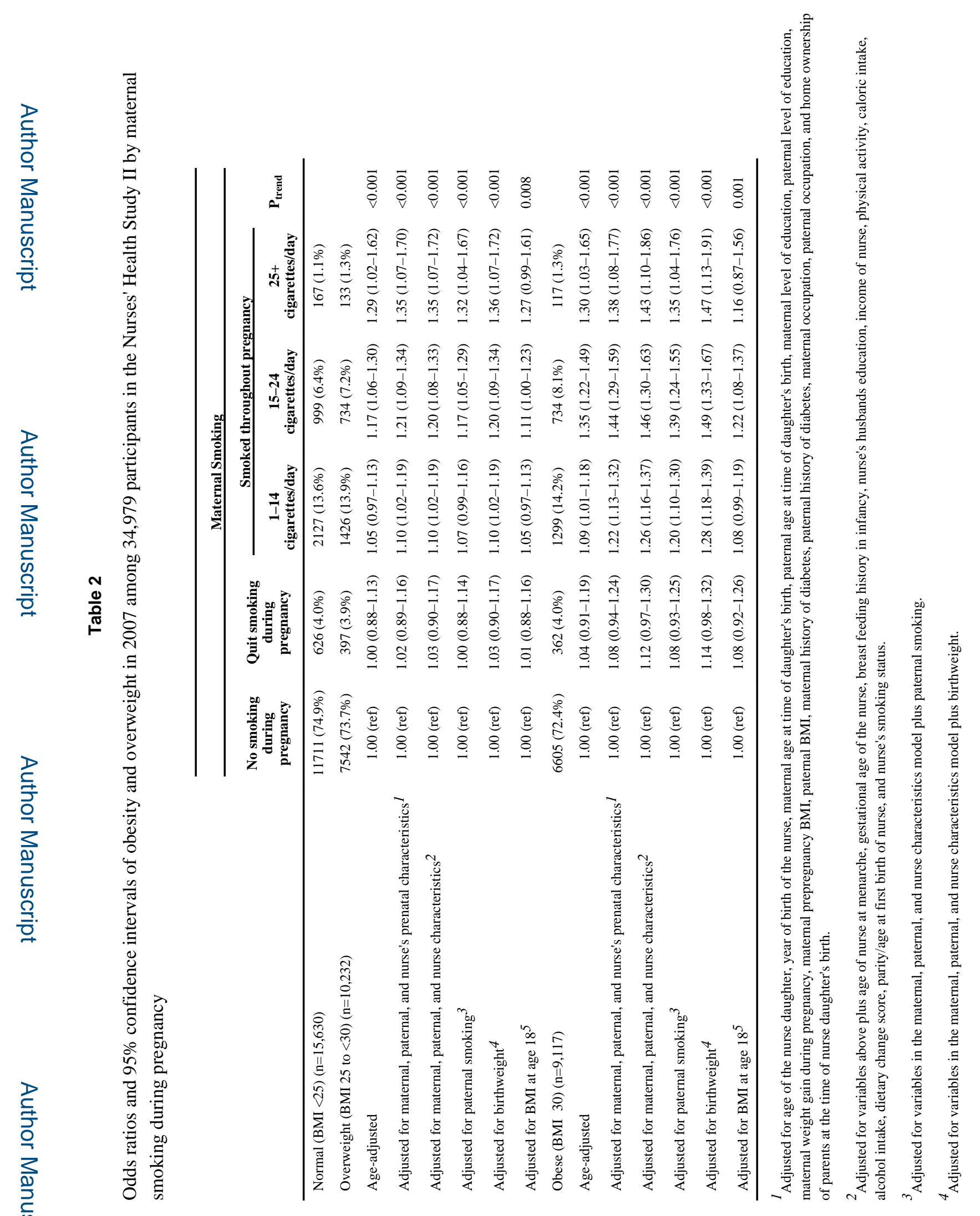

Int J Obes (Lond). Author manuscript; available in PMC 2014 April 01. 


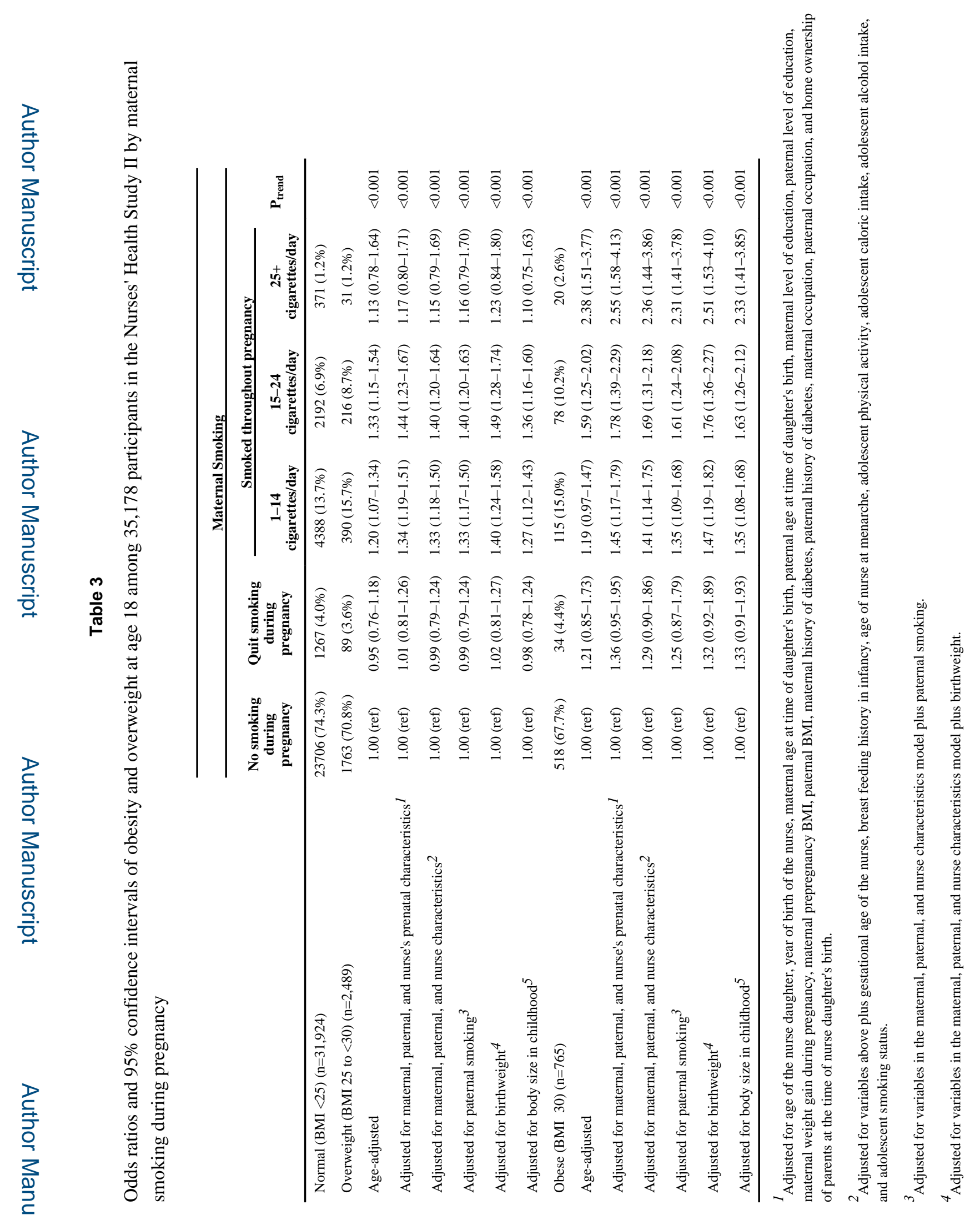

Int J Obes (Lond). Author manuscript; available in PMC 2014 April 01. 


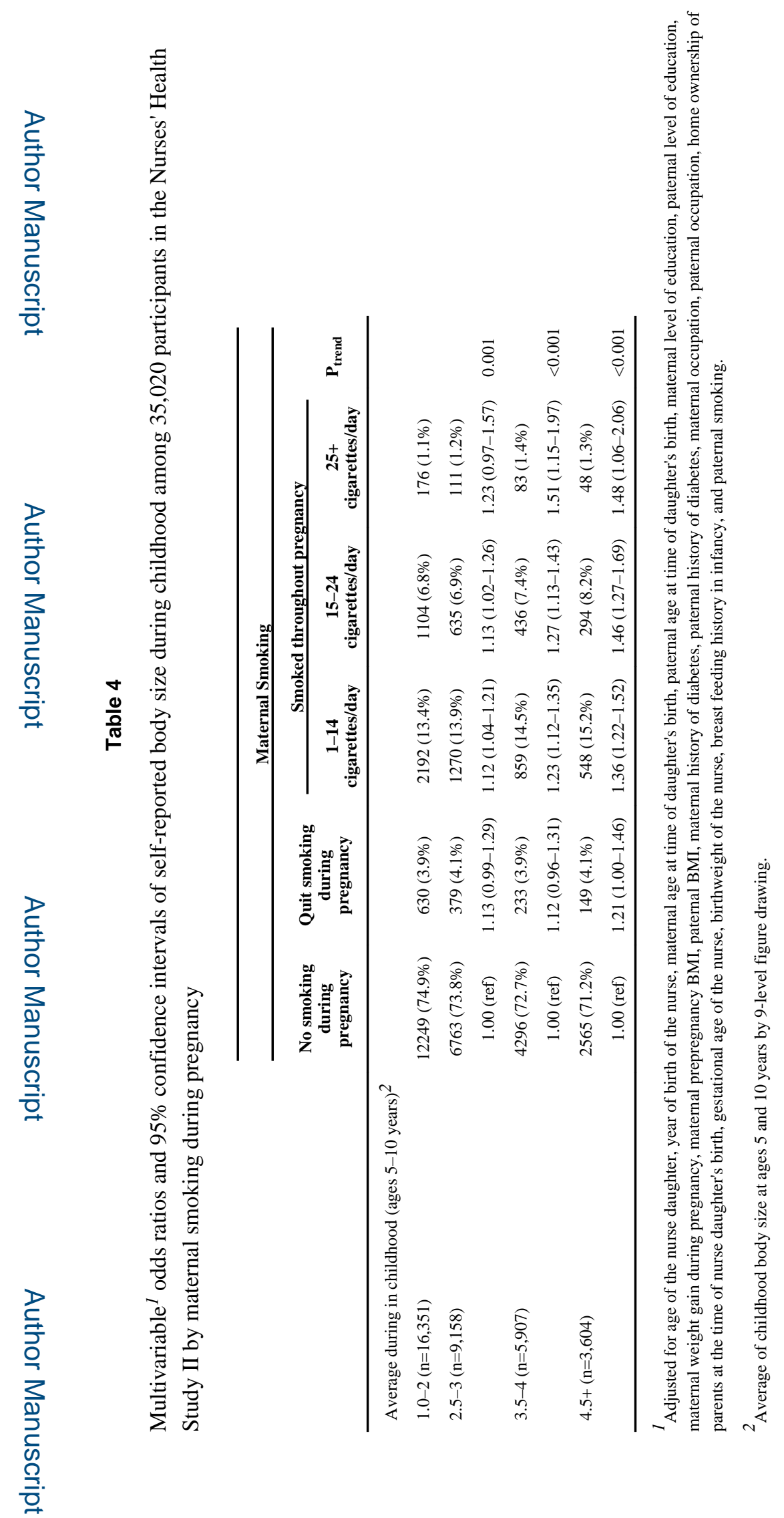

Int J Obes (Lond). Author manuscript; available in PMC 2014 April 01. 


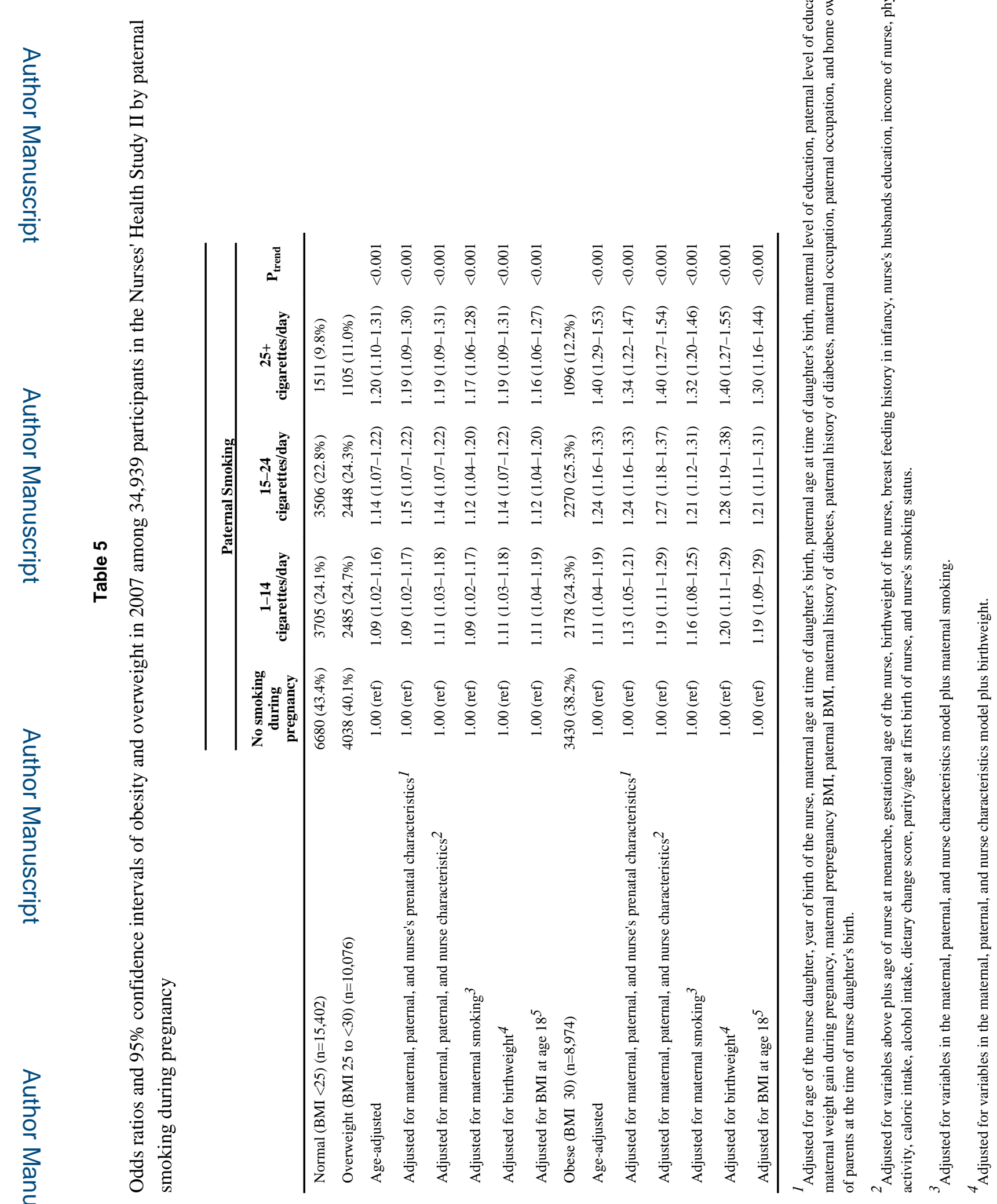

Int J Obes (Lond). Author manuscript; available in PMC 2014 April 01. 
